\title{
Circuit
}

Musiques contemporaines

\section{La Chapelle historique du Bon-Pasteur, Guy Soucie et la création musicale}

\section{Anne Marie Messier}

Volume 24, numéro 2, 2014

URI : https://id.erudit.org/iderudit/1026186ar

DOI : https://doi.org/10.7202/1026186ar

Aller au sommaire du numéro

Éditeur(s)

Les Presses de l’Université de Montréal

ISSN

1183-1693 (imprimé)

1488-9692 (numérique)

Découvrir la revue

Citer cet article

Messier, A. M. (2014). La Chapelle historique du Bon-Pasteur, Guy Soucie et la création musicale. Circuit, 24(2), 89-92. https://doi.org/10.7202/1026186ar d'utilisation que vous pouvez consulter en ligne.

https://apropos.erudit.org/fr/usagers/politique-dutilisation/ 


\section{ACTUALITÉS}

\section{La Chapelle historique du Bon-Pasteur, Guy Soucie et la création musicale}

Anne Marie Messier

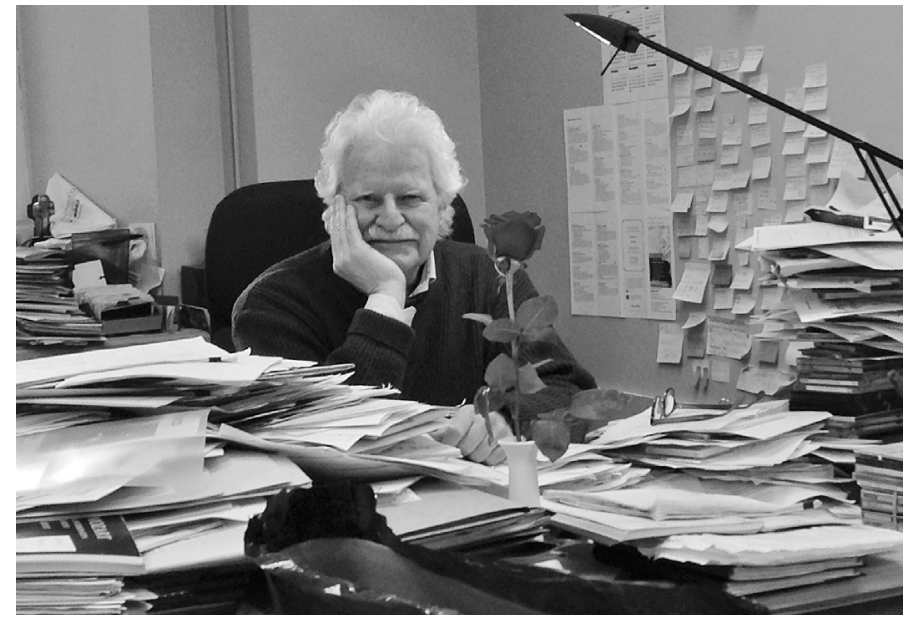

En février 2014, le directeur de la Chapelle historique du Bon-Pasteur à Montréal, Guy Soucie, prenait sa retraite après avoir été aux commandes de la Chapelle depuis sa fondation en 1988. On ne le verra plus aussi souvent assis au jubé, dans le premier fauteuil près de la colonne. Coïncidant avec les 25 ans de la salle de concert, ce départ a suscité plusieurs concerts-hommages et plusieurs articles. On y a rappelé l'inauguration, en septembre 1988 par Marc-André Hamelin, de ce lieu unique dans le microcosme musical montréalais de l'époque; la controverse autour du coût de l'acquisition du piano Fazioli; la feuille de route impressionnante de ces nombreux concerts toutes les semaines, produits en dépit d'un gel du budget de 250000 \$ depuis 15 ans; le maintien de la gratuité des concerts permettant une large démocratisation de la musique; la fidélité d’un public plutôt vieillissant, mais qui se rajeunit lors des concerts de musique contempo- raine. En rencontrant Guy Soucie le 19 février dernier dans la cuisine de la Chapelle historique, l'objectif était surtout de comprendre comment et pourquoi il avait décidé d'engager la Chapelle dans le soutien à la création musicale.

Après l'acquisition de la Chapelle historique, la Ville de Montréal avait fait un appel de projets auquel Guy Soucie, alors directeur de la Maison de la culture Notre-Dame-de-Grâce, a répondu avec enthousiasme. Son projet consacrait la Chapelle à la musique de chambre classique, contemporaine et au jazz, avec trois ou quatre concerts par semaine. Ainsi fut fait. Pour Soucie, la question de présenter de la musique d'aujourd'hui ne se posait même pas. Cela allait de soi, tant avec des concerts étiquetés comme tels que dans le cadre des concerts plus classiques. Il a toujours insisté pour que les artistes incluent une pièce de musique québécoise, montréalaise de préférence. Le désir de 
faire une vraie place à la musique d'aujourd'hui s'est aussi manifesté dans les choix des ensembles en résidence, un programme en place depuis 1997, particulièrement avec le Trio Fibonacci (de 2004 à 2007) et avec l'Ensemble Transmission (depuis 2013).

Hésitant à distinguer les concerts de musique contemporaine les plus réussis, le directeur artistique a tout de même rappelé deux «moments de grâce»: l'interprétation, en 2003 par la pianiste Brigitte Poulin, de For Bunita Marcus (1985) de Morton Feldman, une œuvre exigeante de 77 minutes, une performance qualifiée d'expérience rare par toutes les personnes présentes; ou encore, le cycle Vingt regards sur l'Enfant-Jésus (1944) d'Olivier Messiaen, un véritable cérémonial interprété par la pianiste Louise Bessette en décembre 2008, dans le cadre de l'Automne Messiaen, un événement organisé par l'interprète. Guy Soucie souligne aussi le projet en cours de Louise Bessette: 25 œuvres de 25 compositeurs québécois composées dans les 25 dernières années en quatre concerts, une initiative qui lui fait particulièrement plaisir.

\section{Compositeurs en résidence}

C'est sans conteste la mise en place du programme de compositeur en résidence en 2001 qui a positionné la Chapelle comme un haut lieu de la musique contemporaine à Montréal. Pensée avec le compositeur Simon Bertrand, cette résidence de deux ans installe le créateur dans la vie de la Chapelle: liens fréquents avec le public, participation à la programmation, invitations de solistes et d'ensembles, écriture de nouvelles pièces, travail avec les ensembles en résidence, les interventions du compositeur en résidence sont nombreuses et sur plusieurs plans. Pour la Chapelle, chaque compositeur apporte une vision, une esthétique et un nouveau réseau d'interprètes et de créateurs. Pour chacun des compositeurs, c'est l'annonce d'explorations, de visibilité et de créations à un rythme particulièrement intense. Questionnés sur l'effet le plus marquant de la résidence sur leur parcours, voici ce qu'ont répondu les six compositeurs dans des courriels reçus entre le 26 mars et le 2 mai 2014.

\section{Simon Bertrand, premier compositeur en résidence de 2001 à 2003}

Lorsque je suis rentré au Québec, en 2001, après avoir passé 12 ans à l'étranger, en France et au Japon, je me devais de faire connaître mon travail de compositeur, car, quand j'avais quitté le pays en 1989, le milieu musical me connaissait seulement en tant qu'instrumentiste. Inutile de dire alors l'impact majeur qu'a pu avoir ma résidence à la Chapelle de 2001 à 2003 , pour me faire connaître comme compositeur au Québec. D'autre part, grâce à des collaborations avec des ensembles comme le Trio Contrastes, le duo Traces, l'Ensemble Nishikawa du Japon, le Nouvel Ensemble Moderne (NEM), Quasar, le Quatuor Alcan, l'Orchestre baroque de Montréal (овM), et aussi des solistes remarquables issus de ces ensembles, j'ai pu apprendre mon métier, développer mon esthétique musicale et aussi apprendre à présenter mes œuvres au public. Ainsi, ma résidence à la Chapelle a profondément marqué le développement de ma carrière de compositeur, et je tiens à remercier Guy Soucie d'avoir accepté, à la suite de ma proposition, de relever ce défi, lorsqu'en 2001, nous avions ensemble décidé de créer et structurer ce poste pour la première fois. Rien ne fait plus plaisir que de voir que le poste existe toujours, plus de 10 ans plus tard, et que mes collègues compositeurs aient pu aussi en bénéficier.

\section{Nicolas Gilbert, compositeur en résidence de 2003 à 2005}

J'avais 23 ans au moment de ma nomination comme compositeur en résidence à la Chapelle historique du Bon-Pasteur. La résidence a été, pour moi, le début de bien des choses, notamment de collaborations avec un grand nombre de musiciens de Montréal et d'ailleurs, 
des collaborations qui, dans plusieurs cas, se poursuivent 10 ans plus tard. J'ai composé énormément, pendant ces deux années; au sortir du conservatoire, c'était la façon idéale de poursuivre mon apprentissage. Plusieurs des œuvres issues de la résidence sont encore jouées aujourd'hui; elles constituent, en quelque sorte, la base de mon catalogue. En somme, la résidence m'a servi d'incubateur et m'a permis de m'intégrer extrêmement rapidement à la communauté montréalaise de la musique contemporaine.

\section{Paul Frehner, compositeur en résidence de 2005 à 2007}

Ce fut une période remarquable dans ma carrière de compositeur. J'avais tout juste terminé mes études à l'Université McGill, et cette résidence me donnait l'occasion de me mettre au travail tout de suite et d'écrire de la musique à temps plein. Il s'agissait d'un luxe incroyable. Chaque année, j'étais responsable de la programmation d'une saison de cinq ou six concerts et j'ai eu carte blanche pour inviter une variété de solistes et d'ensembles avec lesquels je cherchais à collaborer. Le projet dont je suis le plus fier, c'est ma pièce pour piano Finnegans Quarks Revival, que j'ai composée pour Brigitte Poulin. J'avais déjà commencé à travailler à ce projet avant ma résidence et peu à peu, tout au long de mon séjour, j’ai pu l'élargir et le développer dans une grande forme en huit mouvements d'une durée de 35 minutes. La résidence m’a donné le temps nécessaire pour laisser mijoter mes idées pour cette pièce et les développer naturellement au fil du temps. Peut-être l'effet le plus remarquable que cette résidence a eu sur moi a été la confiance que cela m’a procurée dans ma propre vision artistique comme compositeur. Au cours des deux années, j’ai écrit beaucoup de musique pour une grande variété d'ensembles de chambre et de solistes et le calendrier était chargé. Grâce au processus, j'en suis sorti avec un sentiment plus fort de mon identité artistique.

\section{Michel Frigon, compositeur en résidence de 2007 à 2009}

L'effet le plus marqué de la résidence sur mon parcours s'est manifesté dans les pièces que j’ai écrites par la suite. De facture plus personnelle, elles sont le fruit des expériences menées en collaboration avec les ensembles et solistes avec qui j’ai travaillé. Nous avons développé ensemble une notation où les phrases, détachées de toute métrique apparente, favorisent un jeu plus intuitif et naturel. Une grande part du succès de la résidence est attribuable aux nombreuses occasions de réunir tous les acteurs d'un concert (compositeurs, interprètes et public) lors de séances pré-concert ou lors d'activités de médiation pour discuter de questions très pointues, comme la relation entre l'idée abstraite, la partition, l'interprète et l'auditoire. C'est une façon très conviviale de démocratiser ce style musical encore perçu par plusieurs comme étant hermétique.

\section{Cléo Palacio-Quintin, compositrice en résidence de 2009 à 2011}

Pour moi, ces deux saisons artistiques de résidence ont certainement contribué à obtenir une plus grande reconnaissance professionnelle et à donner un élan à ma carrière. La visibilité de mon œuvre a été décuplée par le nombre de concerts que j’y ai présentés: 16 concerts, où l'on a présenté 21 de mes œuvres, dont 12 créations. Ma deuxième saison m'a d'ailleurs permis de remporter le prix Opus de compositeur de l'année. Mais au-delà de la visibilité et de la reconnaissance, cette expérience a surtout été extrêmement enrichissante pour plusieurs aspects de mon travail artistique, et non seulement sur le plan de la création comme telle. La possibilité de programmer plusieurs concerts complets m’a permis de développer mon sens de la direction artistique. Je me suis découvert une réelle passion pour partager avec le public ma vision de la musique nouvelle, et ce, en présentant non seulement mes propres œuvres, mais en partageant la scène 
avec mes collègues compositeurs et interprètes (en plus des miennes, 21 créations ont été présentées pendant ma série de concerts). Ces multiples collaborations musicales mont permis de tisser des liens professionnels et des complicités artistiques durables, qui auront une répercussion sur toute ma carrière.

\section{Maxime McKinley, compositeur en résidence de 2011 à 2014}

Au moment d'écrire ces quelques mots, ma résidence n’est pas terminée, donc je n'ai sans doute pas encore le recul nécessaire pour répondre adéquatement à cette question. Cela dit, je pense que cette résidence m’aura beaucoup marqué par sa logique grégaire, axée sur le participatif et le collectif. En effet, la Chapelle est un lieu privilégié de musique de chambre, et c'est bien comme une sorte de «chambriste » - au sens large du terme - qu'est invité à agir, me semblet-il, son compositeur résident, prenant sa place en en faisant aux autres, dans un seul et même mouvement. Lapprofondissement de cet esprit «chambriste» - et donc de la portée du mot «ensemble» (ce «mot-tente», disait joliment Paul Celan) - aura peut-être été l'effet le plus marquant et inspirant de cette résidence sur mon parcours. Cela s'est pratiqué dans la continuité de la résidence, dans une ouverture internationale constante, en composant pour de nombreux musiciens, en programmant et rencontrant des compositeurs de toutes les générations, ou encore, en dialoguant avec des mélomanes de tout acabit. La structure de cette résidence favorise la sociabilité, de manière telle que non seulement le compositeur, mais aussi les interprètes et le public peuvent en tirer profit, isolément et ensemble; comme en musique de chambre, quand la magie opère.

\section{Pour la suite des choses}

Guy Soucie part heureux, avec le sentiment d'avoir réellement contribué à l'évolution de la musique au Québec. Le milieu de la musique l'a bien reconnu en lui décernant, à sa très grande surprise, le prix Opus 2008-2009 du directeur artistique de l'année. Il faut rappeler qu'en 2008, l'administration de la ville de Montréal avait menacé de fermer la Chapelle, comme elle l'avait déjà tenté en 2000. En 24 heures, le milieu musical s'était mobilisé et les protestations ont été si nombreuses et efficaces que la Ville n'a eu d'autre choix que de reculer.

Une des caractéristiques de la Chapelle historique du Bon-Pasteur est que le public doit passer par la scène pour aller dans la salle. On côtoie les instruments, les partitions, les interprètes. La loge des artistes donne directement dans le hall. Ces contacts créent une complicité entre public et musiciens, favorisant une proximité qui va jusqu'à la musique elle-même. Les dialogues qui en découlent, lors de discussions impromptues ou lors des nombreuses présentations d'œuvres, contribuent à l'évolution des goûts musicaux et favorisent l'appropriation des langages musicaux québécois. En multipliant ces occasions, la Chapelle historique du Bon-Pasteur fait figure de modèle à suivre. Guy Soucie peut partir tranquille. Le sillon tracé il y a 25 ans est devenu une bonne route incontournable, et nous sommes nombreux à vouloir en protéger l'accessibilité. 